\title{
Corrigendum: Polariton condensation in solitonic gap states in a one-dimensional periodic potential
}

D. Tanese, H. Flayac, D. Solnyshkov, A. Amo, A. Lemaître, E. Galopin, R. Braive, P. Senellart, I. Sagnes,

G. Malpuech \& J. Bloch

Nature Communications 4:1749 doi: 10.1038/ncomms2760 (2013); Published 23 Apr 2013; Updated 27 Aug 2013

In this Article, there are errors in the labelling of the $x$ axes in Fig. 3. The labels ' -2 ' and ' 2 ' in panels b- $d$ should have been ' -20 ' and ' 20 ', respectively. The correct version of this figure appears below.
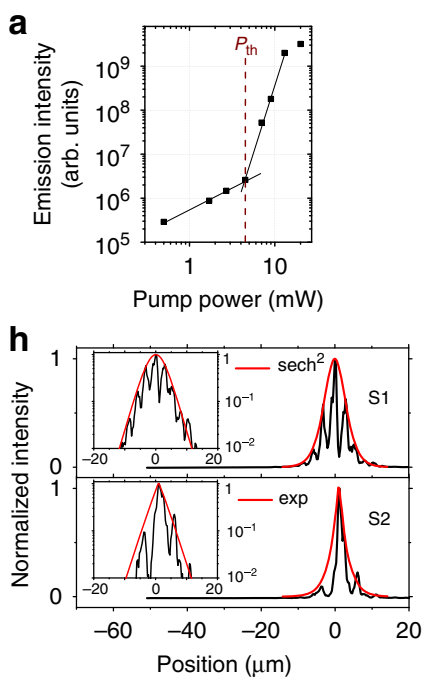

b

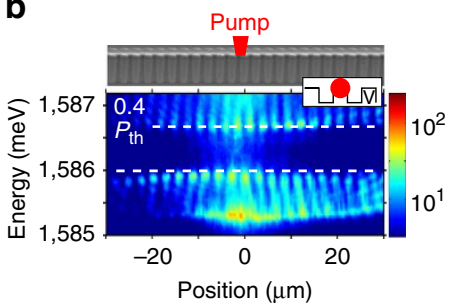

e

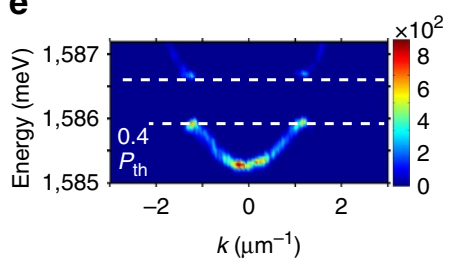

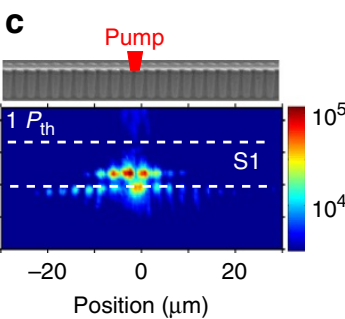

f

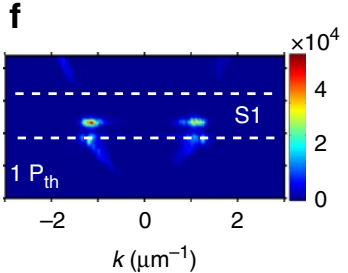

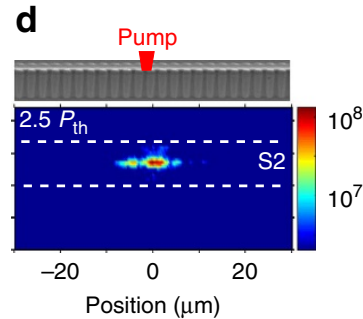

g

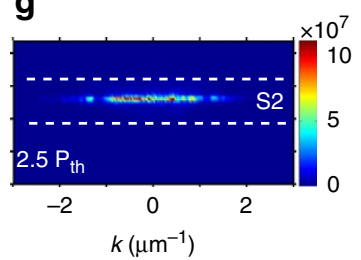

